\title{
El proceso de escritura en la mención de inglés (Educación Primaria)
}

\section{Teaching writing in English major (Primary Education)}

\author{
ANA MARÍA PÉREZ CABELLO \\ ORCID: https://orcid.org/0000-0001-9650-9730 \\ Universidad de Sevilla \\ Departamento de Didáctica de la Lengua \\ y Literatura y Filologías Integradas \\ aperez40@us.es \\ Fecha de recepción: 02-05-2019 \\ Fecha de aceptación: 15-07-2019 \\ DOI: http://dx.doi.org/10.12795/9788447221912.051 \\ Pp.: 1178-1202
}




\section{Resumen}

Este artículo presenta el trabajo investigativo realizado con los estudiantes de la mención de inglés del Grado de Educación Primaria de la Facultad de Ciencias de la Educación de la Universidad de Sevilla. El objetivo principal es evidenciar el proceso de escritura en lengua inglesa tomando como referencia el propio modelo nativo. Mediante actividades que recogen los conocimientos (conceptuales, actitudinales y procedimentales) previos la docente plantea el mapa de contenidos que se va aplicar. Los resultados señalan una importante evolución del aprendizaje en todos los aspectos, así como las bases de una trayectoria profesional próxima.

\section{Abstract}

This paper presents the investigative work carried out with students at the mention of English in the degree of Primary Education in the School of Educational Sciences in the University of Seville. The main objective is to demonstrate the process of writing in the English language with reference to the native model. Through the collection of previous knowledge (conceptual, attitudinal and procedural), the teacher models a map of contents that is applied. The results point to an evolution in learning in all aspects, as well as the grounds for a close professional activity.

Palabras clave: Conocimiento y Uso de la Lengua Inglesa, Educación Primaria, docencia universitaria, experimentación docente universitaria, proceso de escritura.

Key words: Knowledge and Use of English Language, Primary Education, university teaching, university teaching experimentation, writing process.

Jornadas de Formación e Innovación Docente del Profesorado | № 2 (2019) Esta obra se distribuye con la licencia Creative Commons Reconocimiento-NoComercial-SinObraDerivada 
El trabajo que se presenta recoge el resultado de una innovación experimentada Conocimiento y Uso de la Lengua, asignatura de cuarto de la mención de lengua inglesa del grado de Educación Primaria. Las sesiones de prácticas comienzan con actividades generales que implican la oralidad y la didáctica de lenguas (lanzar la pelota para que digan verbos irregulares dada la forma base, preguntas generales sobre algún tema de actualidad, ...). En este ciclo se va a tratar el proceso de escritura en lengua inglesa. Ese es el gran problema del que derivan dos: el proceso de escritura per se y los errores escritos en lengua inglesa. Este tema es bastante complicado pues, en realidad, engloba dos grandes cuestiones que pueden convertirse en un lastre en la vida académica y profesional del estudiantado. Por una parte, encontramos la macroestructura: el diseño de un formato escrito (lo cual no implica que no pueda trabajarse la producción oral); de otro, la microestructura, cómo se organiza el contenido. en inglés cubriendo un espacio de $1 \mathrm{~h} 10 \mathrm{~m}$. cada semana en la sesión práctica.

\section{Diseño previo del CIMA}

El diseño previo del ciclo de mejora incluye el mapa de contenidos y problemas describiendo los logros deseables y los contenidos nucleares que articulan las sesiones de la materia el proceso de escritura en lengua inglesa; el modelo metodológico; la secuenciación de las actividades; el cuestionario inicial y el final (de Alba y Porlán, 2017).

Jornadas de Formación e Innovación Docente del Profesorado | № 2 (2019) Esta obra se distribuye con la licencia Creative Commons 
ANA MARÍA PÉREZ CABELLO

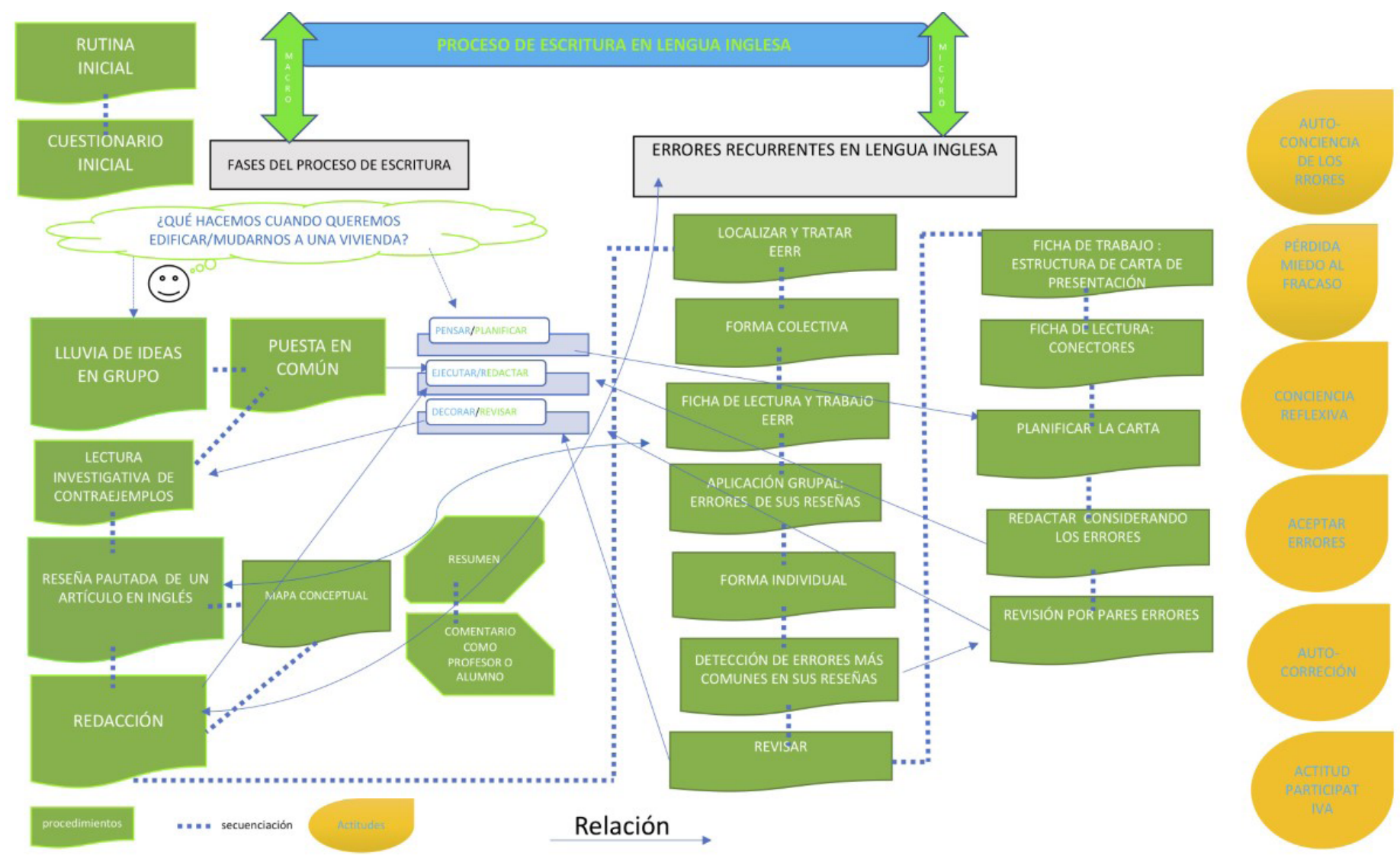

Figura 1. Mapa de contenidos y problemas

Jornadas de Formación e Innovación Docente del Profesorado | № 2 (2019)

(c) (i) $\odot-2$ Esta obra se distribuye con la licencia Creative Commons

Internacional (CC BY-NC-ND 4.0.) 
Tabla 1. Secuenciación de la primera sesión

\section{Proceso de escritura en lengua inglesa \\ Primera sesión (rutinas iniciales)}

Rutina inicial

(5')

\begin{tabular}{l|l|}
$\begin{array}{l}\text { Cuestionario } \\
\text { inicial (15') }\end{array}$ & $\begin{array}{l}\text { Los alumnos realizan el cuestionario para conocer sus } \\
\text { ideas previas sobre los contenidos. }\end{array}$ \\
\hline $\begin{array}{l}\text { Pregunta } \\
\text { inicial }\end{array}$ & $\begin{array}{l}\text { "¿Qué hacemos cuando queremos edificar/mudarnos a } \\
\text { una vivienda?" }\end{array}$ \\
\hline $\begin{array}{l}\text { Ejercicio 1 } \\
\left(20^{\prime}\right)\end{array}$ & $\begin{array}{l}\text { Lluvia de ideas en grupos de 4-6. Los grupos se han } \\
\text { organizado según las partes del discurso que han sacado } \\
\text { de una bolsa: formas de un verbo irregular, conectores, } \\
\text { hipónimos, ... (asi se ha recordado el material anterior.) }\end{array}$ \\
\hline
\end{tabular}

Puesta en común. Llegamos a la conclusión que se ve Ejercicio 2

$\left(15^{\prime}\right)$ en el mapa de contenidos. En él se representa los pasos del proceso de escritura o habitabilidad de una vivienda en colores distintos (pensar, ejecutar, decorar/pensar, redactar, revisar).

Ejercicio 3

$\left(15^{\prime}\right)$
Lectura investigativa de contraejemplos. Primero se analizan ejemplos en el grupo clase. Llevan como tarea analizar el resto en casa.

Jornadas de Formación e Innovación Docente del Profesorado | № 2 (2019) 
Tabla 2. Secuenciación de la segunda sesión

\section{Proceso de escritura en lengua inglesa Segunda sesión}

\begin{tabular}{|c|c|}
\hline $\begin{array}{l}\text { Rutina inicial } \\
\left(10^{\prime}\right)\end{array}$ & $\begin{array}{l}\text { - Se escribe la fecha de forma plena } \\
\text { - Se plantean los puntos principales de la sesión. } \\
\text { - Se anota en la pizarra una frase hecha incompleta } \\
\text { “___-_ speak louder____._Se pretende motivarlos } \\
\text { para que pregunten o investiguen sobre esta oración. }\end{array}$ \\
\hline $\begin{array}{l}\text { Ejercicio } 4 \\
\left(10^{\prime}\right)\end{array}$ & $\begin{array}{l}\text { Se comenta la tarea en grupo, y después, de modo } \\
\text { general. }\end{array}$ \\
\hline $\begin{array}{l}\text { Ejercicio } 5 \\
\left(30^{\prime}\right)\end{array}$ & $\begin{array}{l}\text { Se presenta la herramienta Cmaps. Se les pide que } \\
\text { transfieran las ideas del debate a un mapa conceptual } \\
\text { en base al esquema del proceso de escritura. Trabajan } \\
\text { en grupos formados aleatoriamente. Los alumnos } \\
\text { envían los mapas a la profesora que proyecta algunos } \\
\text { de los más interesantes. Se comentan de forma general } \\
\text { y se extraen principios relevantes. }\end{array}$ \\
\hline $\begin{array}{l}\text { Ejercicio } 6 \\
\left(15^{\prime}\right)\end{array}$ & $\begin{array}{l}\text { Se les explica la tarea que deben realizar de forma } \\
\text { individual, la reseña crítica de un artículo: } \\
\text { - } \text { Seleccionar uno de los dos artículos ofrecidos. } \\
\text { - Realizar un mapa conceptual que recoja las ideas } \\
\text { principales. } \\
\text { - Componer un párrafo que recoja las ideas expresadas } \\
\text { gráficamente. } \\
\text { - Realizar el mismo proceso con los aspectos positivos } \\
\text { o negativos desde la perspectiva o bien como profe- } \\
\text { sor o bien como alumno (ellos están en esa tesitura: } \\
\text { aprendiendo cómo enseñar algo que ellos mismos es- } \\
\text { tán aprendiendo). } \\
\text { Añadir un párrafo introductorio con los datos biblio- } \\
\text { gráficos del artículo. }\end{array}$ \\
\hline
\end{tabular}

Jornadas de Formación e Innovación Docente del Profesorado | № 2 (2019) Esta obra se distribuye con la licencia Creative Commons Reconocimiento-NoComercial-SinObraDerivada Internacional (CC BY-NC-ND 4.0.) 


\section{Tabla 3. Secuenciación de la tercera sesión}

\begin{tabular}{|c|c|}
\hline & $\begin{array}{c}\text { Proceso de escritura en lengua inglesa } \\
\text { Tercera sesión }\end{array}$ \\
\hline $\begin{array}{l}\text { Rutina inicial } \\
\left(10^{\prime}\right)\end{array}$ & $\begin{array}{l}\text { - Se escribe la fecha de forma plena. } \\
\text { - Se plantean los puntos principales de la sesión. } \\
\text { - La profesora lanza una pelota y solicita conectores de } \\
\text { los alumnos. Es uno de los errores recurrentes en sus } \\
\text { composiciones. }\end{array}$ \\
\hline Ejercicio 7 (15') & $\begin{array}{l}\text { Se presenta un texto con los errores más comunes } \\
\text { detectados en la corrección de las reseñas. Se lee de } \\
\text { forma extensiva. Se muestran algunos contraejemplos. } \\
\text { Se les pide una versión correcta justificando su } \\
\text { respuesta. }\end{array}$ \\
\hline $\begin{array}{l}\text { Ejercicio } 8 \\
\left(25^{\prime}\right)\end{array}$ & $\begin{array}{l}\text { Trabajan el resto de los ejemplos en grupo. En este caso } \\
\text { aunque se han agrupado ellos mismos la profesora } \\
\text { asigna un número y un papel a cada miembro. No } \\
\text { realizar la tarea puede tener repercusiones negativas } \\
\text { en su calificación de prácticas. Se les conciencia de } \\
\text { que esta es una tarea de prácticas lo que ocurre que } \\
\text { se desarrolla en clase en vez de fuera. Estas son las } \\
\text { responsabilidades: } \\
\text { - números } 1 \text { son el capitán del silencio. Moderan el } \\
\text { tono de voz de su grupo. } \\
\text { - números } 2 \text { averiguan que cada uno cumple con su } \\
\text { responsabilidad. } \\
\text { - números } 3 \text { manejan el ordenador. } \\
\text { - números } 4 \text { son el portavoz. }\end{array}$ \\
\hline Tarea (10') & $\begin{array}{l}\text { Se explica la tarea para la semana siguiente: fichas de } \\
\text { lectura y fichas de trabajo sobre educación y rasgos de } \\
\text { personalidad. }\end{array}$ \\
\hline
\end{tabular}

Jornadas de Formación e Innovación Docente del Profesorado I № 2 (2019) 
Tabla 4. Secuenciación de la cuarta sesión

\begin{tabular}{|c|c|}
\hline \multicolumn{2}{|r|}{ Cuarta sesión } \\
\hline $\begin{array}{l}\text { Rutina inicial } \\
\left(5^{\prime}\right)\end{array}$ & $\begin{array}{l}\text { - La profesora escribe la fecha de forma errónea. Es- } \\
\text { pera que alguien se dé cuenta y lo comunique. } \\
\text { - Se plantean los puntos principales de la sesión. }\end{array}$ \\
\hline Ejercicio 9 (20') & $\begin{array}{l}\text { Cada grupo se encarga de presentar la corrección del } \\
\text { contraejemplo asignado. }\end{array}$ \\
\hline Ejercicio 10 (10') & $\begin{array}{l}\text { Se pide voluntarios para que hablen de su } \\
\text { personalidad y/o bagaje académico. Asi pueden } \\
\text { poner en práctica las fichas de trabajo que tenían } \\
\text { como tarea. Las fichas son tarea de la semana } \\
\text { anterior y tienen las respuestas en la plataforma. Se } \\
\text { suben dos días antes de la clase. }\end{array}$ \\
\hline Ejercicio 11 (15') & $\begin{array}{l}\text { Se presenta una carta formal que está mal diseñada } \\
\text { tanto en formato como en contenido. Se discuten las } \\
\text { carencias principales en estos dos aspectos. Se les } \\
\text { pide que cuando expresen sus ideas usen conectores } \\
\text { del tipo regarding, concerning, as far as _-_- is } \\
\text { concerned). Se recuerda su uso con algunos ejemplos. }\end{array}$ \\
\hline Tarea $\left(20^{\prime}\right)$ & $\begin{array}{l}\text { Se explica la tarea. Se les indican unas páginas donde } \\
\text { pueden encontrar anuncios reales ofertando puestos } \\
\text { de trabajo relacionados con educación. Deben elegir } \\
\text { uno y escribir una carta de presentación, cover } \\
\text { letter. Deben autocorregirse la carta siguiendo las } \\
\text { fichas de lectura y trabajo, así como y la aplicación } \\
\text { Grammarly. De otro lado, se les enseña la posibilidad } \\
\text { de insertar la carta en la aplicación: https://europass. } \\
\text { cedefop.europa.eu/editors/en/cl/compose. Deben } \\
\text { entregar un portfolio de estas sesiones que recoja: } \\
\text { mapa conceptual del contenido de la carta, borrador } \\
\text { inicial, comentario de los errores cometidos, versión } \\
\text { mejorada de Grammarly, y la versión final. }\end{array}$ \\
\hline
\end{tabular}

Jornadas de Formación e Innovación Docente del Profesorado | № 2 (2019) Esta obra se distribuye con la licencia Creative Commons Reconocimiento-NoComercial-SinObraDerivada Internacional (CC BY-NC-ND 4.0.) 


\section{Aplicación del CIMA}

Primeramente, se va a presentar el relato de la ejecución del ciclo de mejora. A continuación, se realizar una comparativa entre los resultados del cuestionario inicial y el final.

\section{Primera sesión}

Rutina inicial (5'). Se pide a un alumno que escriba la fecha de forma plena. Se lanza la pelota y se les pide conectores. Se muestran expectantes ante la presencia de la pelota.

Cuestionario inicial (15'). Los alumnos realizan el cuestionario para conocer sus ideas previas sobre los contenidos. En vista de que las cuestiones exploratorias se han realizado inmediatamente antes de iniciar el tema, de hecho, en la misma sesión, no ha dado tiempo de remodelar las actividades planteadas. Teniendo ya un mapa de contenidos de esta parte de la asignatura, será más fácil con posterioridad remodelar las actividades. Sin embargo, pasaría el cuestionario antes de iniciar el tema para poder adaptar las actividades y desarrollarlas ya adaptadas desde el primer momento.

Ejercicio 1. (20'). Lluvia de ideas en grupos de 4-6 para responder la pregunta los grupos se han organizado según las partes del discurso que han sacado de una bolsa: formas de un verbo irregular, conectores, hipónimos, ... (así se ha recordado el material anterior).

\section{Segunda sesión}

Rutina inicial (10'). Se escribe la fecha. Se plantean los puntos principales de la sesión. Se anota en la pizarra una frase hecha incompleta "_____- speak louder_____. Se 
pretende motivarlos para que pregunten o investiguen sobre esta oración.

Ejercicio 4 (10'). Se comenta la tarea en grupo, y después, de modo general. Como se ha comentado antes, trabajar primero en grupos, y posteriormente, en gran grupo hace la clase muy rentable porque como sentencia Gamo (2016) "El cerebro necesita emocionarse para aprender" (citado en Torres Menárguez, 2016). Además, se muestran satisfechos y de desarrollan la competencia social, mayoritariamente las relaciones interpersonales (Mora, 2013, Mora 2017). Si no han preparado la tarea, el trabajo en grupo puede ayudarles a ponerse en día, aunque no se debe usar siempre para que no caigan en la rutina de no hacer realizar las actividades. Se les pregunta por la forma en que se ha llevado la actividad. Los comentarios son positivos; argumentan que es una reflexión en voz alta. Actitudinalmente, toman conciencia de la importancia de escucharse a ellos mismos y a otros. Agradecen que sea una lectura comentada entre todos. Por otra parte, les aporta libertad al ser distinta a otras actividades más directivas.

Ejercicio 5 (30'). Se presenta la herramienta Cmaps. Se les pide que transfieran las ideas del debate a un mapa conceptual en base al esquema del proceso de escritura. Trabajan en grupos formados aleatoriamente. Los alumnos envían los mapas a la profesora que proyecta algunos de los más interesantes. Se comentan de forma general y se extraen principios relevantes.

Esta actividad debería haber sido más extensa porque los estudiantes no parecían tener muy claro lo que era un buen mapa conceptual. Se les indica que puede usar otra aplicación como Powerpoint.

Ejercicio $6\left(15^{\prime}\right)$. Se les explica la tarea que deben realizar de forma individual, la reseña crítica de un artículo:

- seleccionar uno de los dos artículos ofrecidos. 
- realizar un mapa conceptual que recoja las ideas principales.

- componer un párrafo que recoja las ideas expresadas gráficamente.

- realizar el mismo proceso con los aspectos positivos o negativos desde la perspectiva o bien como profesor o bien como alumno (ellos están en esa tesitura: aprendiendo cómo enseñar algo que ellos mismos están aprendiendo).

- añadir un párrafo introductorio con los datos bibliográficos del artículo.

Las instrucciones han supuesto en otras ocasiones un obstáculo para la realización adecuada del ejercicio. Dada esta circunstancia se decidió detallarlas en clase. Parecen estar acostumbrados a estar muy dirigidos (recuérdese el comentario anterior). De otro lado, no leen con atención. Suelen focalizarse en el resultado final sin seguir el proceso que se les pide. Ya ocurrió esto en otras ocasiones cuando se les colgaba una tarea en la plataforma. Es el caso de la reseña es aún más necesario asegurarse pues es un formato que no conocen en su propia lengua. No se preguntó por este formato de texto en el cuestionario inicial ya que el docente ha impartido la materia de Lengua Española en el tercer curso; sin embargo, se hará la próxima vez. Como tarea que es no parecen estar muy contentos. Al estar tan detallado el proceso la ven muy extensa. Se les anima diciendo que es parte de una tarea puntuable que además les ayudará a prepararse el examen de la asignatura.

\section{Tercera sesión}

Rutina inicial (10'). Se escribe la fecha. Se plantean los puntos principales de la sesión. La profesora lanza una pelota y solicita conectores de los alumnos. Es uno de los errores recurrentes en sus composiciones. Es encantador 
ver sus caras están esperando que se les lance la pelota mientras el docente la sostiene.

Ejercicio 7 (15'). Se presenta un texto con los errores más comunes detectados en la corrección de las reseñas. Se lee de forma extensiva. Se muestran algunos contraejemplos. Se les pide que den una versión correcta justificando su respuesta. Los ejercicios de análisis son básicos para afianzar los contenidos teóricos.

Ejercicio 8 (25'). Trabajan el resto de los ejemplos en grupo. En este caso, aunque se han agrupado ellos mismos, la profesora asigna un número y un papel a cada miembro. Se les advierte que no realizar la tarea asignada puede tener repercusiones negativas en su calificación de prácticas. Se les conciencia de que esta es una tarea de prácticas que se desarrolla en clase en vez de fuera. Estas son las responsabilidades:

- los números 1 son el capitán del silencio. Deben moderar el tono de voz de su grupo.

- los números 2 deben averiguar que cada uno cumple con su responsabilidad.

- los números 3 manejan el ordenador.

- los números 4 son el portavoz.

El tema de los errores les preocupa. Es dificil determinar por qué el trabajo discurre en un tono de voz correcto, sin alzar la voz: por la repercusión de la tarea para su aprendizaje o porque existe el capitán silencio.

Tarea (10'). Se explica la tarea para la semana siguiente: fichas de lectura y fichas de trabajo sobre educación y personalidad. 


\section{Cuarta sesión}

Rutina inicial (5'). La profesora escribe la fecha de forma errónea. Espera que alguien se dé cuenta y lo comunique. Se plantean los puntos principales de la sesión.

Ejercicio $9\left(20^{\prime}\right)$. Cada grupo se encarga de presentar la corrección del contraejemplo asignado en la sesión anterior. Lo hacen muy bien.

Ejercicio $10\left(10^{\prime}\right)$. Se pide voluntarios para que hablen de su personalidad y/o bagaje académico. Así pueden poner en práctica las expresiones de las fichas de trabajo que tenían como tarea. Las fichas son tarea de la semana anterior y tienen las respuestas en la plataforma. Se suben dos días antes de la clase. Son muchos los que quieren intervenir.

Ejercicio $11\left(15^{\prime}\right)$. Se presenta una carta formal que está mal diseñada tanto en formato como en contenido. Se discuten las carencias principales en estos dos aspectos. Se les pide que cuando expresen sus ideas usen conectores del tipo regarding, concerning, as far as _-_ is concerned). Se recuerda su uso con algunos ejemplos. Como siempre, siguiendo las pautas didácticas de la profesora, se fomenta la oralidad. Verbalizando el pensamiento se activa la memoria en la lengua extranjera y se recupera información y estrategias que creíamos no conocer. Escucharse a sí mismo incentiva el autoconocimiento y el control sobre la materia, como ha quedado dicho antes. Si los alumnos no se emplean en la tentativa de hablar nunca lo harán. Este aspecto es fundamental en la formación del alumnado de cara a su trabajo. Ellos suelen comentar que no sienten pudor ante los colegiales, aunque sí ante los adultos. Consiguientemente. Necesitan entrenar su subcompetencia productiva oral de cara a la defensa de futuros trabajos académicos, oposiciones e incluso entrevistas con los padres. En las primeras situaciones la inhibición se 
duplica: además del miedo a hablar en público se une el temor a hablar en una lengua extranjera. Para evitar que siempre tomen la palabra los mismos alumnos la profesora indica el nombre del alumno y le invita a comentar sus ideas. Saben que es importante la participación en las prácticas y que además tiene peso en la calificación de esta parte.

Tarea $\left(20^{\prime}\right)$. Se explica la tarea. Se les indican una página donde pueden encontrar anuncios reales que ofertan puestos de trabajo relacionados con educación. Deben elegir uno y escribir una carta de presentación, cover letter. Deben autocorregirse la carta siguiendo las fichas de lectura y trabajo, así como y la aplicación Grammarly. De otro lado, se les enseña la posibilidad de insertarla carta en la aplicación: https://europass.cedefop.europa.eu/editors/en/cl/compose. Deben entregar un portfolio de estas sesiones que recoja: mapa conceptual del contenido de la carta, borrador inicial, comentario de los errores cometidos, versión mejorada de Grammarly, y la versión final. Se muestran muy entusiasmados con la idea. El resultado de aprendizaje no solo le es útil a corto plazo para aprobar la materia sino además en el futuro. Hay un alumno que conoce el editor online europass. Se le invita a presentarlo a los demás. Es una forma de reconocer el conocimiento que tiene y de hacer ver a los demás que tienen mucho que dar a la sociedad.

Pasemos a ver los resultados comparativos de los ambos cuestionarios. La primera pregunta (¿Cuáles son tus principales dificultades cuando escribes en español?) pretende comprobar la actitud del estudiante ante su competencia escritora en español (todos tienen como primera lengua el español). Resulta llamativo que no 5 alumnos o sean conscientes de tener errores. Es importante subsanar estos por lo que se tomaron medidas al respecto creando pequeños seminarios y tutorías grupales. 


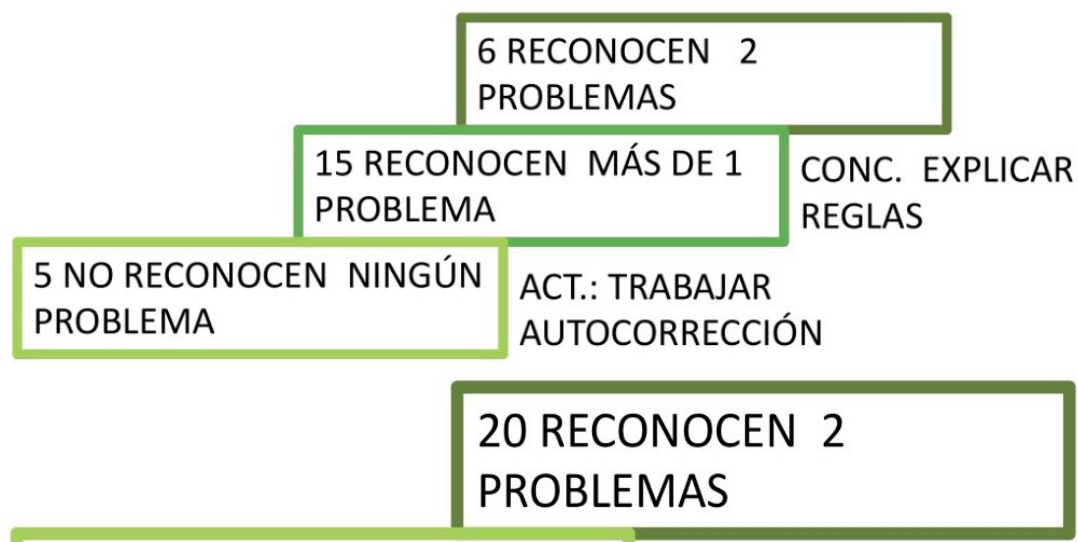

\section{RECONOCEN 1 PROBLEMA}

Figura 3. Escalera de apren. cuest. 1, preg. 1

F igura 4. Escalera de apren. cuest. 2, preg. 1

En la comparativa la cantidad es inversamente proporcional a la cualidad del aprendizaje actitudinal pues son capaces de la autocrítica.

También se desarrolla un estudio de las dificultades apuntadas por los estudiantes. Nos ayuda en la selección de contenido que luego tendrán que autocorregirse. Se comparan las dificultades en la primera y segunda lengua incidiendo en la corrección de los errores más comunes.

Tabla 5. Dificultades en español

\begin{tabular}{|c|c|}
\hline Signos de puntuación & 5 \\
\hline Tiempos verbales & 1 \\
\hline Ortografia & 5 \\
\hline Sinónimos & 5 \\
\hline Falta de vocbulario culto & 5 \\
\hline Acentuación & 1 \\
\hline Expresión & 4 \\
\hline Ninguna & 5 \\
\hline
\end{tabular}

Jornadas de Formación e Innovación Docente del Profesorado | № 2 (2019) Esta obra se distribuye con la licencia Creative Commons Reconocimiento-NoComercial-SinObraDerivada Internacional (CC BY-NC-ND 4.0.) 
La segunda cuestión (Especifica los problemas que encuentras cuando escribes en inglés) pide que se concreten los problemas al escribir en inglés. Coincide además que estos alumnos tienen una buena destreza oral adquirida mediante el aprendizaje informal (relación con nativos, estancias en países anglófonos, por ejemplo), lo que, por experiencia con mis hijos, les cuesta pulir su detareza escrita a través del aprendizaje más ortodoxo.

Tabla 6. Dificultades en inglés

\begin{tabular}{|c|c|}
\hline Uso de tiempo verbales & $\begin{array}{l}\text { Formas compuestas } 8 \\
\text { Pasados irregulares } 6 \\
\text { Condicional } 3 \\
\text { Presente simple } 3 \\
\text { Presente continuo } 2\end{array}$ \\
\hline Uso de gerundios/participios & $\begin{array}{l}\text { Gerundios } 9 \\
\text { Participios irregulares } 9\end{array}$ \\
\hline Uso de preposiciones & $\begin{array}{l}\text { Uso general } 8 \\
\text { Uso específico con verbos } 15\end{array}$ \\
\hline Uso de conectores & Uso general 14 \\
\hline Falta de vocabulario & $\begin{array}{l}\text { Básico } 6 \\
\text { Específico } 13 \\
\text { Sinónimos } 3\end{array}$ \\
\hline Uso de signos de puntuación & $\begin{array}{l}\text { Puntos y comas } 5 \\
\text { Comas } 3\end{array}$ \\
\hline Otros & Expresión 1 \\
\hline Ninguno & 3 \\
\hline
\end{tabular}

\section{RECONOCEN 1 PROBLEMAS}

\section{RECONOCEN 2 PROBLEMA}

\section{RECONOCEN 3 PROBLEMA}

Figura 5. Escalera de apren. cuest. 1, preg. 2

Jornadas de Formación e Innovación Docente del Profesorado | № 2 (2019) Esta obra se distribuye con la licencia Creative Commons Reconocimiento-NoComercial-SinObraDerivada Internacional (CC BY-NC-ND 4.0.) 


\author{
1 EMPLEA UN MÉTODO \\ COMPLETO \\ 2 EMPLEAN DOS FASES PROC.: CENTRARNOS EN LA \\ DE UN MÉTODO \\ 3 EMPLEAN UNA FASE PROC.: CENTRARNOS EN LA \\ DE UN MÉTODO \\ SEGUNDA FASE, REDACTAR \\ 3 EMPLEAN UN PROC.: CENTRARNOS EN LA PRIMERA \\ BORRADOR \\ FASE, PLANIFICAR Y ORGANIZAR \\ 7 ORGANIZAN PROC.: PROFUNDIZAR EN LAS FASES \\ LIBREMENTE \\ DEL PROCESO DE ESCRITURAS \\ 10 ESCRIBEN \\ DIRECTAMENTE \\ PROC.: COMPARAR TEXTOS ESCRITOS \\ Y NO ESCRITOS CON UN PROCESO
}

Figura 6. Escalera de apren. cuest. 2, preg. 2

Se aprecia cómo aquellos que nos reconcocen algún problema han desaparecido y aumentan el número de alumnos con menos problemas.

\title{
6 EMPLEA UN MÉTODO COMPLETO
}

\section{EMPLEAN DOS FASES DE UN MÉTODO}

\section{EMPLEAN UNA FASE DE UN MÉTODO}

Figura 7. Escalera de apren. cuest. 1, preg. 3

Jornadas de Formación e Innovación Docente del Profesorado | № 2 (2019) Esta obra se distribuye con la licencia Creative Commons Reconocimiento-NoComercial-SinObraDerivada 4.0 Internacional (CC BY-NC-ND 4.0.) 


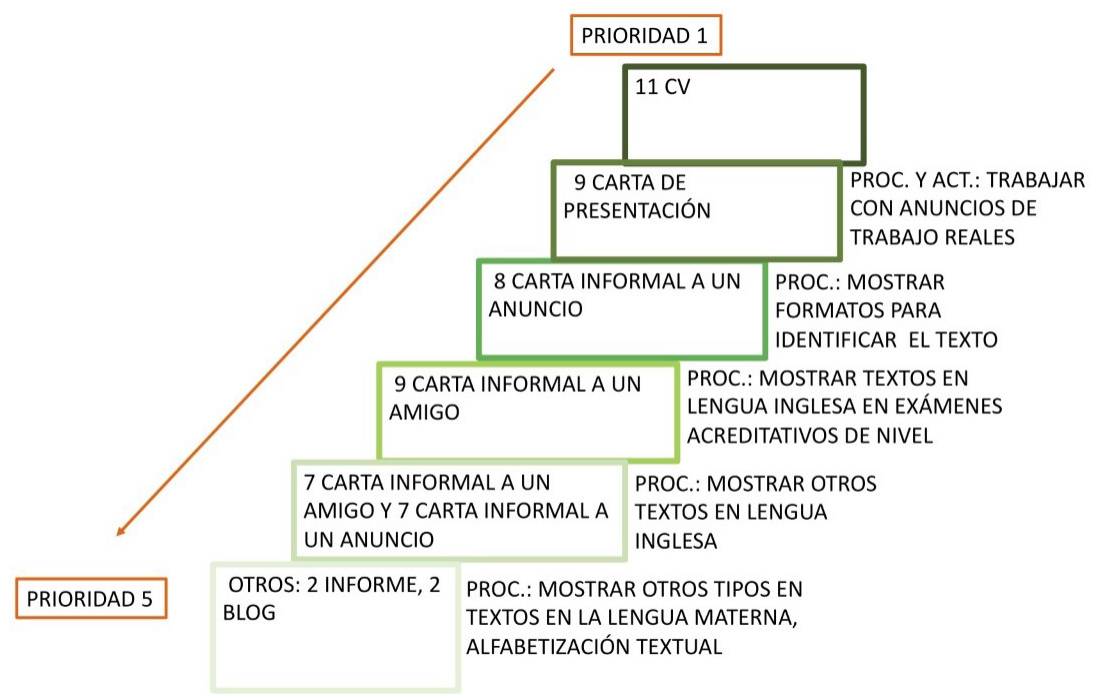

Figura 8. Escalera de apren. cuest. 2, preg. 3

La última pregunta de este cuestionario (¿Qué documento encuentras más útil? Ordénalos de 1 a 5 según tu prioridad (1 mayor prioridad, 5 menor prioridad).) se dedica a averiguar tanto las necesidades del alumnado como su conocimiento del mundo profesional. Siguiendo los datos detallados de la tabla de análisis, la escalera se realiza en base a los documentos que quedan en las diferentes posiciones, de 1 a 5, como se puede apreciar.

Tabla 7. Prioridad de tipología textual

\begin{tabular}{|l|l|l|l|l|l|}
\hline 1 & $11 \mathrm{CV}$ & $\begin{array}{l}12 \text { Carta de } \\
\text { presentación }\end{array}$ & $\begin{array}{l}\text { 2 Carta } \\
\text { informal a } \\
\text { un amigo }\end{array}$ & 1 Anuncio & \\
\hline 2 & $\begin{array}{l}\text { 9 Carta de } \\
\text { presentación }\end{array}$ & $\begin{array}{l}\text { 8 Carta } \\
\text { informal a un } \\
\text { anunciante }\end{array}$ & $4 \mathrm{CV}$ & $\begin{array}{l}3 \text { Carta } \\
\text { informal a un } \\
\text { amigo }\end{array}$ & 2 Anuncio \\
\hline 3 & $\begin{array}{l}8 \text { Carta } \\
\text { informal a un } \\
\text { anunciante }\end{array}$ & 7 Anuncio & $\begin{array}{l}6 \text { Carta } \\
\text { informal a } \\
\text { un amigo }\end{array}$ & $3 \mathrm{CV}$ & $\begin{array}{l}\text { 2 Carta de } \\
\text { presentación }\end{array}$ \\
\hline
\end{tabular}

Jornadas de Formación e Innovación Docente del Profesorado | № 2 (2019) Esta obra se distribuye con la licencia Creative Commons Reconocimiento-NoComercial-SinObraDerivada Internacional (CC BY-NC-ND 4.0.) 


\begin{tabular}{|l|l|l|l|l|l|}
\hline 4 & $\begin{array}{l}\text { 9 Carta } \\
\text { informal a un } \\
\text { amigo }\end{array}$ & $\begin{array}{l}\text { 7 Carta } \\
\text { informal a un } \\
\text { anunciante }\end{array}$ & 5 Anuncio & $3 \mathrm{CV}$ & $\begin{array}{l}\text { 2 Carta de } \\
\text { presentación }\end{array}$ \\
\hline 5 & $\begin{array}{l}7 \text { Carta } \\
\text { informal a un } \\
\text { amigo }\end{array}$ & $\begin{array}{l}\text { 7 Carta } \\
\text { informal a un } \\
\text { anunciante }\end{array}$ & 6 Anuncio & $\begin{array}{l}\text { 4 Carta de } \\
\text { presentación }\end{array}$ & 2 CV \\
\hline OTROS & 2 Informe & 2 Blog & & & \\
\hline
\end{tabular}

Es interesante ver cómo en la primera ocasión algunos consideran la carta informal a un amigo más importante que la redacción del propio currículum o la carta de presentación. Por otra parte, llama la atención cómo el mercantilismo en el que vivimos y la fugacidad del empleo condicionan que el pensamiento de los estudiantes se enfoque en el día a día sin contemplar la posibilidad de consolidar un plan de futuro. Se evidencia en la priorización de una carta informal a un anunciante o la elaboración de un anuncio sobre una vivienda respecto a textos destinados a abrirse camino en la profesión para la que se preparan. Es llamativo que dos alumnos no entienda (uno de ellos de los más brillantes) a la actividad en el cuestionartio inicial pues, simplemente, se limitan a enumerar las posibilidades de 1 a 5.

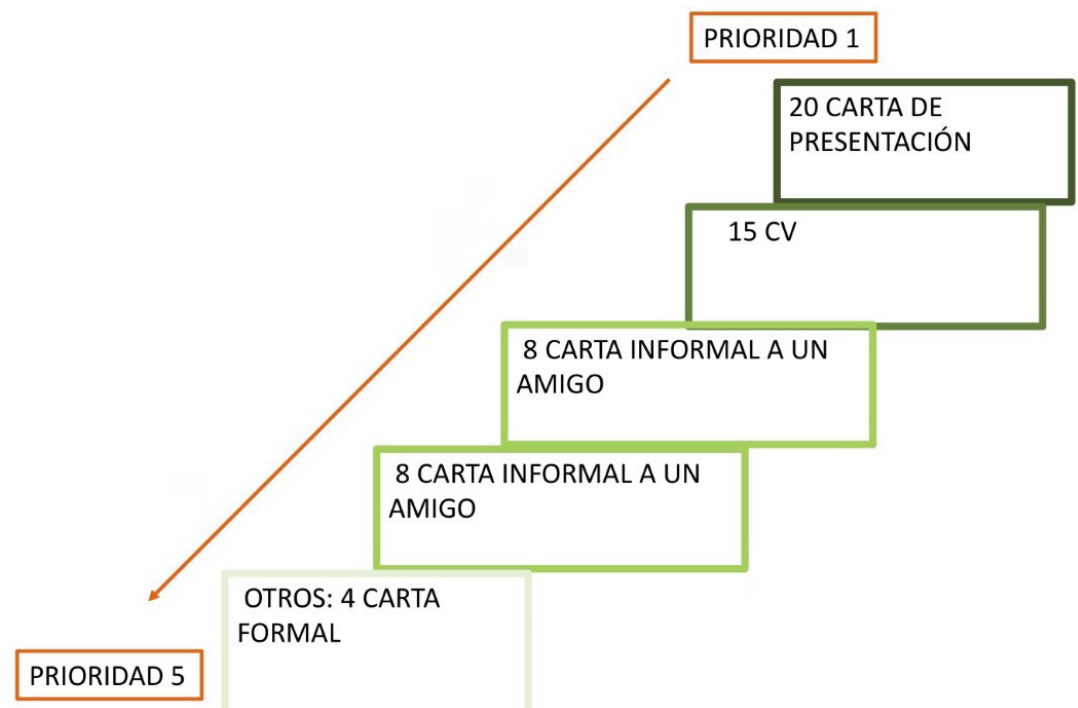

Figura 9. Escalera de apren. cuest. 1, preg. 4

Jornadas de Formación e Innovación Docente del Profesorado | № 2 (2019) Esta obra se distribuye con la licencia Creative Commons Reconocimiento-NoComercial-SinObraDerivada Internacional (CC BY-NC-ND 4.0.) 
La carta de presentación no es un documento no es requerido en nuestra cultura; sin embargo, gracias al trabajo en clase con anuncios reales dentro del entorno anglosajón se observa un cambio de opinión situando la carta de presentación en primera posición. En el segundo cuestionario añaden en Otros la cara formal sin ser conscientes que la carta de presentación ya lo es. Hay que seguir trabajando este aspecto.

\section{Evaluación del CMD}

El aspecto más importante en relación al aprendizaje ha sido retomar la funcionalidad de los modelos mentales (Rivero y Porlán, 2017así como la autorreflexión del profesor (Porlán 2008). Había trabajado con los schemata en inglés durante la elaboración de mi tesis (Didáctica de la Literatura en Segundas Lenguas). Aunque sí los había tratado desde una perspectiva constructivista nunca lo había hecho desde una personal. Dicho de otro modo, el curso me ha hecho abogar por el desarrollo personal del alumno no solo intelectual. Así explicita Bain (2007) la importancia del mismo cuando se refiere al pensamiento de Norden, "cada disciplina puede encontrar vías de [para] enfrentar a los estudiantes con preguntas sobre su identidad como seres humanos"' (citado en Bain 2007, p. 106). De otro lado, aunque siempre he considerado las ideas previas del alumnado nunca lo había hecho de esta forma. Bain (2007) y Porlán (2017) nos reconducen a personalizar el aprendizaje gracias a la consideración de los modelos mentales y la organización de tales modelos. Yo usaba el símil del ascenso a una montaña para explicar la evolución del aprendizaje individual pero la metáfora de la escalera es más coherente y permite visualizar mejor los problemas que plantea subir los escalones y la complejización del conocimiento. Durante su carrera docente ha pretendido centrar 
el aprendizaje en el alumnado. Aun así, la comprensión y trabajo con los modelos mentales formulados en el curso son herramientas fundamentales para diseñar un aprendizaje centrado y trabajado desde el estudiante.

Siempre, también, la docente ha valorado positivamente la interacción con el alumno, no solo conmigo sino entre ellos. De ahí que la organización de contenidos a raíz de las preguntas guía o planteamientos de problemas sociales o disciplinares vertebre la interacción en diferentes direcciones. En este sentido, los contenidos se transforman en una dinámica fluida de trabajo. Los mapas de contenido permiten interrelacionarlos y jerarquizarlos. En este sentido, dado que los contenidos ahora son organizados desde una perspectiva distinta las secuencias de actividades son más pautadas y completas. Al desagregar los contenidos en centrales y periféricos, y categorizarlos (conceptuales, actitudinales y procedimentales) se establecen objetivos más específicos, lo que se traduce en un aprendizaje procesual no objetivizado por completo. En otras palabras, en un aprendizaje que no responde exclusivamente a objetivos didácticos.

Otro aspecto importante ya señalado parcialmente antes, es el uso de las preguntas clave como eje del proceso de aprendizaje -diría que casi más para el profesor que para el alumno.

No cabe duda de que el modelo lógico-investigativo supone romper los esquemas mentales del profesor en cuanto a su concepción de la enseñanza (Finkel, 2008). Con este método los propósitos de las acciones que se llevan a cabo son claramente identificables: no tanto en cuanto a las propias actividades sino en relación a la selección de contenidos y el diseño de las mismas. Esto nos lleva a otro aspecto relevante, la función del profesor. Este se transforma en un generador de preguntas, una especie de abogado del diablo. El conocimiento del docente es 
tridimensional, como los contenidos adquiridos por los estudiantes. Debe poseer tres clases de conocimiento para que la experiencia intelectual sea fructífera: conocimiento disciplinar, conocimiento didáctico sobre la misma, y conocimiento sobre las fortalezas y debilidades de sus estudiantes. Esta imagen ilustra esta idea,

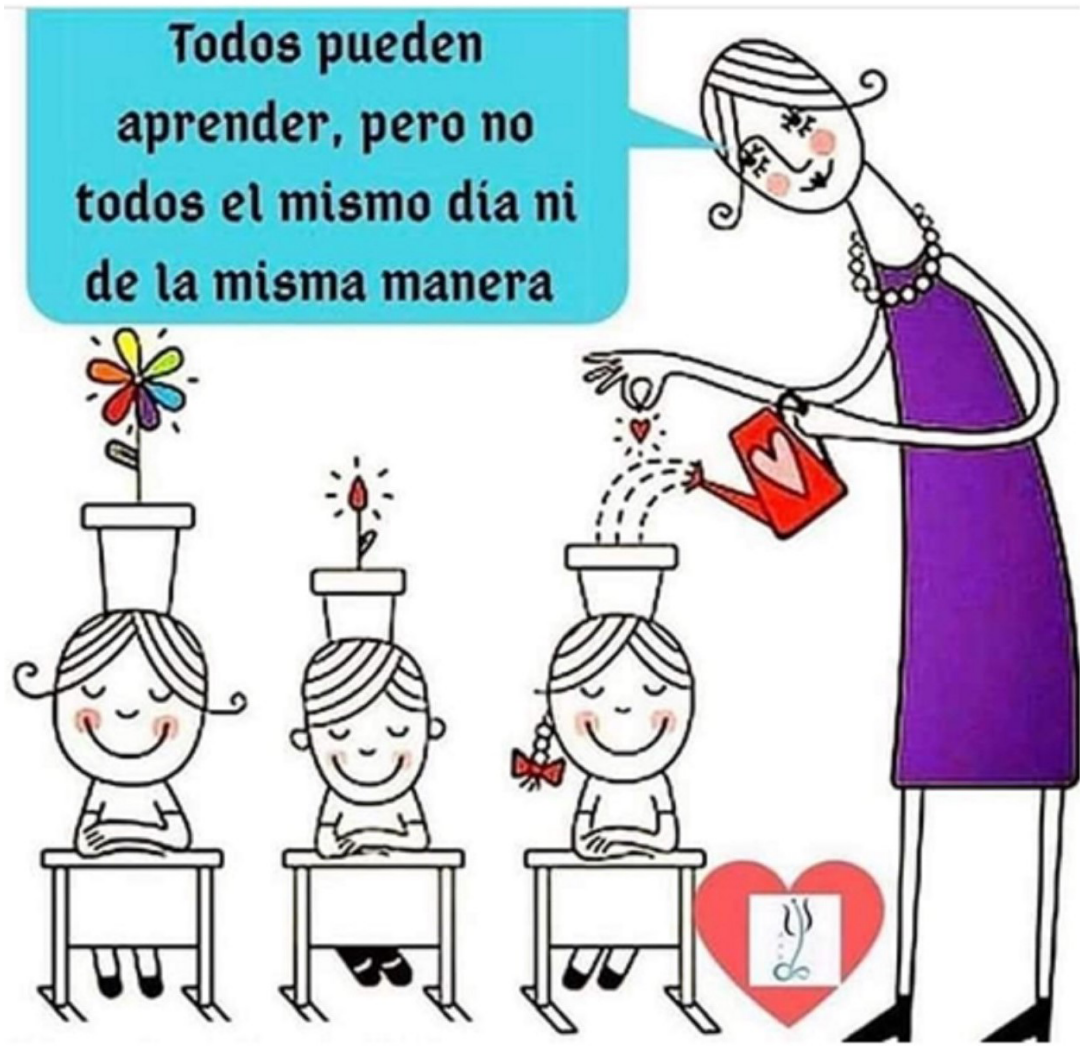

Figura 11. Todos pueden aprender

La evaluación es la tarea más pesada por la objetividad que requiere, aunque el sesgo es inevitable. Por ello, me parecen muy adecuadas las recomendaciones sobre el uso de diferentes instrumentos de evaluación. La elección de los mismos debe configurarse según qué contenidos queremos evaluar. Nunca había usado las fichas de evaluación, pero a partir de ahora las usaré en diferentes 
momentos de la evaluación sumativa para poder comprender hasta qué punto los estudiantes pueden analizar y sintetizar los contenidos. En este sentido, resulta plausible mencionar no solo la calidad de los contenidos sino la tipología de los mismos. Por ello, en la escalera de aprendizaje cada escalón podría constar de diferentes losas para cada tipo de contenido. El uso de las escaleras de aprendizaje ha sido sorprendente en relación a lo que los alumnos conocen sobre el tema que se va a tratar y para comprobar que usan conocimientos de los diferentes tipos señalados. Así lo demuestra el hecho de que al plantear una situación didáctica no conocen el aspecto conceptual, aunque si procedimental ya que usan técnicas didácticas vistas en clase.

La autoevaluación reflexiva de este trabajo nos lleva a unos principios didácticos personales que cierran este artículo:

- Realizar el cuestionario antes de empezar con la unidad para poder así tomar decisiones desde el primer momento (lo trasladaría a una sesión anterior o bien lo haría virtualmente dando un tiempo de ejecución para evitar en la medida de lo posible que no consulten fuentes de información electrónicas o fisicas. Aunque se vuelva a poner en práctica el ciclo de mejora el alumnado y sus circunstancias son distintas, así que el análisis de los cuestionarios y el reajuste de las preguntas son necesarios en cada nueva ocasión.

- La planificación debe comenzar con preguntas que nos sitúen en la realidad del alumno frente a la disciplina. Estas deberían cubrir todos los tipos de contenidos.

- -Según las respuestas de los alumnos se deben escalonar y secuenciar los contenidos.

- -Debemos ser conscientes de la flexibilidad del mapa de contenidos.

- -Debemos ser consecuentes y coherentes en nuestros criterios de evaluación. Por ello cuando diseñemos el 
mapa debemos esbozar al menos cómo evaluaremos y tipificar los resultados de aprendizaje.

- La interacción presencial y virtual debe ser fomentada en todas direcciones.

- La escalera de aprendizaje determina la situación del estudiante y los obstáculos a los que proceso y alumno se enfrentan.

- Las TIC deben responder a las necesidades del alumnado y eficacia del proceso de aprendizaje.

- Incentivar la autorreflexión en voz alta. Así verbalizamos nuestro pensamiento y somos conscientes de lo que sabemos.

- Impulsar las relaciones entre los contenidos.

- Ofrecer la información desde distintos canales para fomentar la sintesis de ideas.

- Recurrir a diferentes tipos de actividades. Ello permite romper las expectativas del alumnado motivando su atención y curiosidad. Es, en definitiva, una forma de atender a los diferentes tipos de inteligencias.

Jornadas de Formación e Innovación Docente del Profesorado | № 2 (2019) Esta obra se distribuye con la licencia Creative Commons 


\section{Bibiliografía}

Bain, K. (2007). Lo que hacen los mejores profesores universitarios. Valencia: Publicaciones de la Universidad de Valencia.

De Alba, N. y Porlán, R. (2017). La metodología de enseñanza. En Porlán (Coord.) Enseñanza universitaria: cómo mejorarla pp. 37-53. Madrid: Morata.

Finkel, D. (2008). Dar clase con la boca cerrada. Valencia: Publicaciones de la Universidad de Valencia.

Gamo, R. (2016) en Torres Menárguez (18 de julio de 2016). El cerebro necesita emocionarse para aprender. El País. Recuperado de https://elpais.com/economia/2016/07/17/actualidad/1468776267 359871.html

Mora, F. (2013). Neuroeducación. Madrid: Alianza.

Mora, F. (2017). en Torres Menárguez (20 de febrero de 2017). "Hay que acabar con el formato de clases de 50 minutos". El País. Recuperado de https://elpais.com/economia/2017/02/17/actualidad/1487331225 284546.html

Porlán, R. (2008). (Universidad de Sevilla) El diario de clase y el análisis de la práctica. Averroes. 1-8. Recuperado de http://www.juntadeandalucia.es/averroes/impe/ web/contenido?pag=/co...tigacionEducativa/Seccion/ InvestigarEnEducacion/T213 diariodeclase

Porlán, R. (Coord.) (2017). Enseñanza universitaria: cómo mejorarla. San Sebastián de los Reyes, Madrid: Morata.

Rivero, A. y Porlán, R. (2017). La evaluación en la enseñanza universitaria en Porlán (Coord.) Enseñanza universitaria: cómo mejorarla (pp. 73-91). Madrid: Morata.

Todos pueden aprender. Recuperado de https://www. facebook.com/Asociacion.Proyecto.Autismo/photo s/a.10152324532533116/10157185879878116/?type $=3 \&-$ theater

Torres Menárguez, A. (18 de julio de 2016). El cerebro necesita emocionarse para aprender. El País. Recuperado de https://elpais.com/economia/2016/07/17/actualidad/1468776267 359871.html

Torres Menárguez, A. (20 de febrero de 2017). "Hay que acabar con elformato de clases de 50 minutos". El País. Recuperado de https://elpais.com/economia/2017/02/17/ actualidad/1487331225 284546.html

Jornadas de Formación e Innovación Docente del Profesorado | № 2 (2019) Esta obra se distribuye con la licencia Creative Commons Reconocimiento-NoComercial-SinObraDerivada Internacional (CC BY-NC-ND 4.0.) 